\title{
Influence du poids à la naissance et du génotype sur la composition chimique de l'agneau nouveau-né
}

\author{
Yseult VILLETTE et B. AUROUSSEAU * \\ avec la collaboration technique de Marinette Martinadd * et J. Leroux \\ I.N.R.A., Laboratoire de la Production ovine, \\ * Laboratoire du Métabolisme énergétique, \\ Centre de Recherches de Clermont-Ferrand, \\ Theix, F 63110 Beaumont
}

\section{Résumé}

La composition chimique de 76 agneaux nouveau-nés appartenant à différentes races (14 Limousine : L, 5 Romanov : R, et 12 Ile-de-France : IDF) ou à leur croisement $(9 \mathrm{R} \delta \times \mathrm{L}$ ㅇ et $36 \mathrm{IDF} \delta \times(\mathrm{R} \times \mathrm{L})$ ㅇ $\mathbf{l}$ a été déterminée et reliée, sauf pour le génotype Romanov (R.), au poids des agneaux à la naissance.

Pour les agneaux L, IDF et IRL, les teneurs en eau $(765 \pm 14 \mathrm{~g} / \mathrm{kg})$, énergie $(1133 \pm 70 \mathrm{kcal} / \mathrm{kg})$ et protéines $(163 \pm 13 \mathrm{~g} / \mathrm{kg})$ sont indépendantes du poids à la naissance. En revanche dans le génotype $R L$, les coefficients d'allométrie sont significativement différents de 1 et prennent pour valeur respective $0,92(\mathrm{P} 2<0,01), 1,28$ ( $\mathrm{P}<0,05)$ et $1,32(\mathrm{P}<0,05)$.

Les teneurs en cendres $(42,7 \pm 4,7 \mathrm{~g} / \mathrm{kg})$ ainsi que celles des différents minéraux analysés (calcium, phosphore, magnésium, sodium et potassium) sont indépendantes du poids à la naissance.

Ces résultats de composition chimique sont discutés en rapport avec les taux de mortalité néonatale observés sur ces mêmes génotypes.

\section{1. - Introduction}

Pour les brebis à forte prolificité, la fin de la gestation et le début de la lactation correspondent à des périodes de besoins élevés de l'ensemble des nutriments; ces besoins peuvent en partie être couverts par l'utilisation des réserves corporelles, principalement à des fins énergétiques. Cependant l'utilisation de ces réserves doit rester limitée pour éviter les troubles du métabolisme ou des réductions excessives du poids à la naissance des agneaux de portées multiples (Tissier \& THERIEZ, 1978), phénomènes susceptibles d'entraîner de fortes mortinatalités (ALEXANDER, 1974). Aussi est-il donc préférable d'assurer au mieux le rationnement de ces brebis fortes productrices, et pour cela, de connaître avec précision leurs besoins pendant la ges- 
tation. Actuellement, il est possible d'évaluer les dépôts journaliers à la fin de la gestation à partir du poids et de la composition chimique du ou des fœetus à terme (I.N.R.A., 1978). Cependant les informations disponibles concernent principalement les génotypes utilisés à l'étranger : agneaux ou fœtus Mérinos (WENINGER, FunK \& Koenig, 1955 ; Langlands \& Sutherland, 1968, 1969) ou issus d'un croisement avec des béliers suffolk (Rattray et al., 1974 ; Mac Donald et al., 1979).

Il paraît donc intéressant de chercher à préciser la composition chimique d’agneaux nouveau-nés dans le cas des génotypes utilisés en France, et d'envisager la description de leurs variations avec la taille des portées dont ils sont issus ou leurs poids à la naissance. Ce dernier point permet en outre d'examiner s'il est possible de relier les différences de mortalité néonatale entre ces génotypes (Houssin-VILLETTE \& BRELURUT, 1980) à des différences de composition corporelle.

\section{2. - Matériel et méthodes}

\section{Animaux}

Les données proviennent de lanalyse de 76 agneaux du troupeau du CRZV de Theix, nés de 1975 à 1977 et morts de cause accidentelle (Dystocie) au moment de la parturition. Les compositions chimiques correspondant à 17 d'entre eux ont fait l'objet d'une première communication (I.N.R.A., 1978).

Trois races : Limousine (L), Romanov (R) ou Ile-de-France (IDF) ainsi que leurs croisements : $\mathrm{R} \hat{o} \times \mathrm{L} q(\mathrm{RL})$ ou $\mathrm{I} \delta(\mathrm{R} \delta \times \mathrm{L} q)$ \& (IRL) sont représentées (tableau 1). A cette diversité des races parentales s'ajoutent celles des saisons d'agnelage et des sexes (tableau 1), ainsi que celle de la taille de la portée : 8 agneaux simples, 31 doubles, 28 triples et 9 quadruples.

Le calcul de l'intervalle entre les dates d'insémination artificielle des brebis et de leur mise-bas a permis de déterminer l'âge gestationnel de 49 agneaux : 90 p. 100 d'entre eux sont nés entre 143 et 149 jours de gestation.

\section{Traitement des échantillons}

Les agneaux sont pesés et identifiés dans les cinq heures suivant la naissance puis conservés en l'état (en particulier sans que les toisons n’aient été séchées) en chambre froide à -20 "C sous enıballage étanche. Ils sont soumis individuellement à un premier broyage dans l'azote liquide à l'aide d’un broyeur à hélice. Environ $700 \mathrm{~g}$ de broyat obtenu, sous forme de poudre congelée, sont soumis à un second broyage qui aboutit à l'obtention d'une pâte très homogène à partir de laquelle sont prélevés, pour analyse, deux échantillons représentatifs de $120 \mathrm{~g}$ environ.

Leur teneur en eau est déterminée par lyophilisation. Les toisons des animaux nayant pas été séchées, les résultats recouvrent non seulement l'eau du corps des agneaux mais aussi l'eau imprégnant leur toison. Par mesure de simplification nous emploierons néanmoins le terme «d'eau corporelle» pour l'exposé des résultats. 


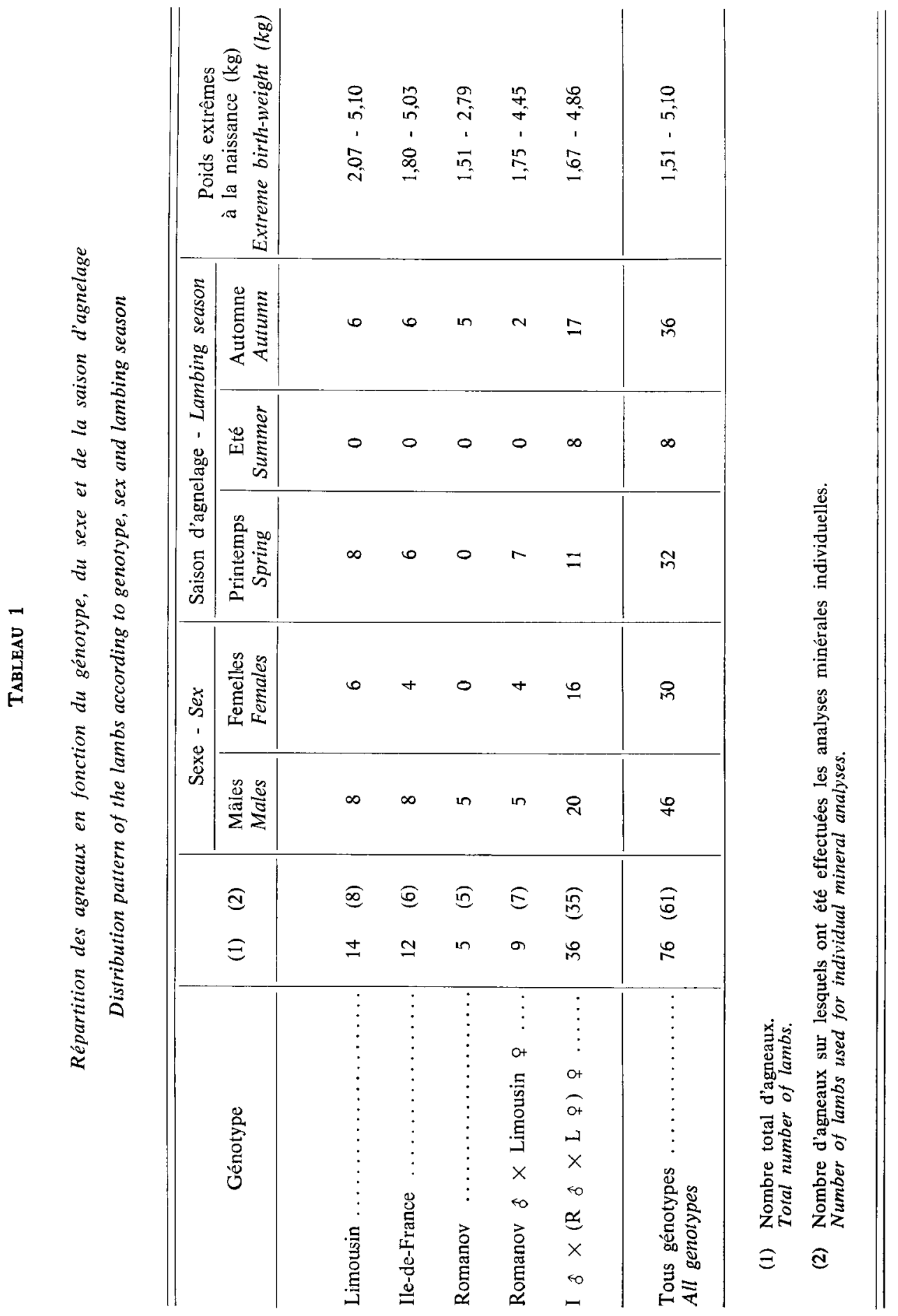


Les étapes suivantes de l'analyse de la composition des échantillons sont effectuées sur les produits lyophilisés, les résultats étant corrigés en fonction de la teneur en matière sèche obtenue au moment des analyses après dessiccation à l'étuve pendant 24 heures à $100^{\circ} \mathrm{C}$.

Les teneurs en azote sont déterminées sur chacun des échantillons selon la méthode KJEldahl, les teneurs en énergie par calorimétrie semi-adiabatique et les teneurs en cendres par incinération au four à 700 " $\mathrm{C}$ pendant 8 heures.

Les teneurs en protéines ont été calculées en affectant le coefficient de 6,25 aux teneurs en azote, et les teneurs en matière organique non protéique par différence entre la teneur en matière sèche et celles en protéines et cendres.

La composition minérale a été étudiée sur 64 agneaux. Le phosphore minéral a été dosé par colorimétrie (KALCKaR, 1947), le calcium et le magnésium par spectrophotométrie d'absorption atomique et le sodium et potassium par émission de flamme (PERKIN ELMER 700).

Les résultats présentés portent sur l'agneau en entier.

\section{Analyses statistiques}

La répartition des agneaux a permis une analyse simultanée des facteurs poids à la naissance et génotype. Cependant les agneaux Romanov, peu nombreux et de poids peu variable n'ont pas été inclus dans ces analyses. Seule la composition chimique moyenne ainsi que les écarts types sont présentés pour cette race. Nous avons utilisé, pour relier les quantités totales d'un élément $Y$ (eau, énergie, protéines...) au poids à la naissance $(P)$, le modèle de relation d'allométrie de HuXley sous sa forme $\operatorname{logarithmique} \log \mathrm{Y}=\mathrm{a}+\mathrm{b} \log \mathrm{P}$. D'après cette notation, la teneur en un élément pour un animal i de poids $\mathrm{Pi}$ à la naissance correspond au rapport $\frac{\mathrm{Yi}}{\mathrm{Pi}}$. Les différences entre génotype des coefficients d'allométrie (b) ou des ordonnées à l'origine (a) ont êtẽ testées par analyse de la covariance. Ces comparaisons nécessitent l'homogénéité des variances résiduelles et les tests effectués (test de BARTLETT) montrent que cette condition n'est pas toujours réalisée (tableaux 2 et 4 ) et l'analyse statistique perd alors de sa précision, les seuils de signification étant surévalués.

Outre l'influence de ces deux facteurs génotype et poids à la naissance, celles éventuelles du sexe ou de la saison d'agnelage ont été recherchées par analyse de variance sur les résidus des analyses statistiques précédentes. Enfin, l'influence de la taille de la portée, indépendamment de son effet sur le poids à la naissance a pu être étudiée sur 35 agneaux IRL par analyse de covariance $(11,15$ et 9 agneaux issus respectivement de portées doubles, triples et quadruples). 


\section{3. - Résultats}

Selon les analyses statistiques, la composition chimique de l'agneau nouveau-né ne semble pas affectée par la taille de la portée, indépendamment de la liaison avec le poids à la naissance. Par ailleurs elle ne paraît pas modifiée par le sexe ou la saison d'agnelage.

Dans ces conditions les résultats rapportés ci-dessous se limitent à la description des variations de la composition chimique de l'agneau nouveau-né en fonction de son poids et de son type génétique.

\section{Teneur en eau de l'agneau nouveau-né}

Les relations d'allométrie entre l'eau corporelle et le poids des agneaux, pour chacun des 3 génotypes : L, IDF et IRL sont voisines. Ni les coefficients d'allométrie (b), ni les ordonnées à l'origine (a) ne montrent de différences significatives (tableau 2). De plus, les coefficients d'allométrie ne diffèrent pas significativement de l'unité, ce qui indique que les quantités d'eau corporelle sont proportionnelles au poids des animaux. Pour ces 3 génotypes les teneurs (Y/P) en eau de l'agneau nouveau-né $(\mathrm{g} / \mathrm{kg})$ sont donc indépendantes de son poids (figure 1a) et du même ordre de grandeur : 777,765 et $760 \mathrm{~g} / \mathrm{kg}$ respectivement pour les agneaux de génotype $\mathrm{L}$, IDF et IRL (tableau 3). Elles présentent par ailleurs des coefficients de variation faibles (moins de 2 p. 100). Enfin les agneaux Romanov (tableau 3) présentent des teneurs moyennes en eau du même ordre : $760 \mathrm{~g} / \mathrm{kg}$ que les animaux des 3 génotypes considérés ci-dessus.

En revanche, les agneaux de type génétique RL présentent un coefficient d'allométrie significativement inférieur à l'unité $(\mathrm{p}<0,01)$ et à ceux des trois autres génotypes $\mathrm{L}$, IDF et IRL $(\mathrm{p}<0,05)$. Ce résultat indique que la teneur en eau $(\mathrm{Y} / \mathrm{P}) \mathrm{de}$ l'agneau diminue quand son poids augmente (figure 1a) et cette allométrie négative aboutit à des teneurs moyennes en eau corporelle inférieures à celles observées pour les autres génotypes (tableau 3) et variant de $777 \mathrm{~g} / \mathrm{kg}$ pour des agneaux pesant $2 \mathrm{~kg}$ à la naissance à $730 \mathrm{~g} / \mathrm{kg}$ pour des agneaux pesant $4 \mathrm{~kg}$.

\section{Teneur en énergie, protéines et matière organique non protéique de l'agneau nouveau-né}

L'énergie et les protéines corporelles des agneaux des 4 génotypes présentent par rapport à leur poids à la naissance une allométrie positive (coefficient d'allométrie supérieur à 1) ce qui indique un accroissement des teneurs (Y/P) en énergie $(\mathrm{kcal} / \mathrm{kg})$ et en protéines $(\mathrm{g} / \mathrm{kg})$ lorsque le poids à la naissance augmente. Cependant les coefficients d'allométrie ne sont significativement différents de l'unité que pour les agneaux de génotype RL (tableau 2) avec des valeurs de 1,28 ( $p<0,05)$ pour l'énergie et $1,32(\mathrm{p}<0,05)$ pour les protéines. Ainsi les teneurs en énergie $(\mathrm{kcal} / \mathrm{kg})$ ou en protéines $(\mathrm{g} / \mathrm{kg}$ ) des agneaux $\mathrm{RL}$ varient de $1065 \mathrm{kcal} / \mathrm{kg}$ ou $160 \mathrm{~g} / \mathrm{kg}$ à $1280 \mathrm{kcal} / \mathrm{kg}$ ou $200 \mathrm{~g} / \mathrm{kg}$ respectivement pour des agneaux pesant de 2 à $4 \mathrm{~kg}$ à la naissance. 


\section{Tableau 2}

Equations d'allométrie reliant les quantités totales $(Y)$ en eau, énergie, protéines et matière organique non protéique du corps de l'agneau nouveau-né à son poids à la naissance $(P)$

Allometry equations between the total quantities $(Y)$ of water, energy, non-protein-organic matter and lamb birth-weight $(P)$

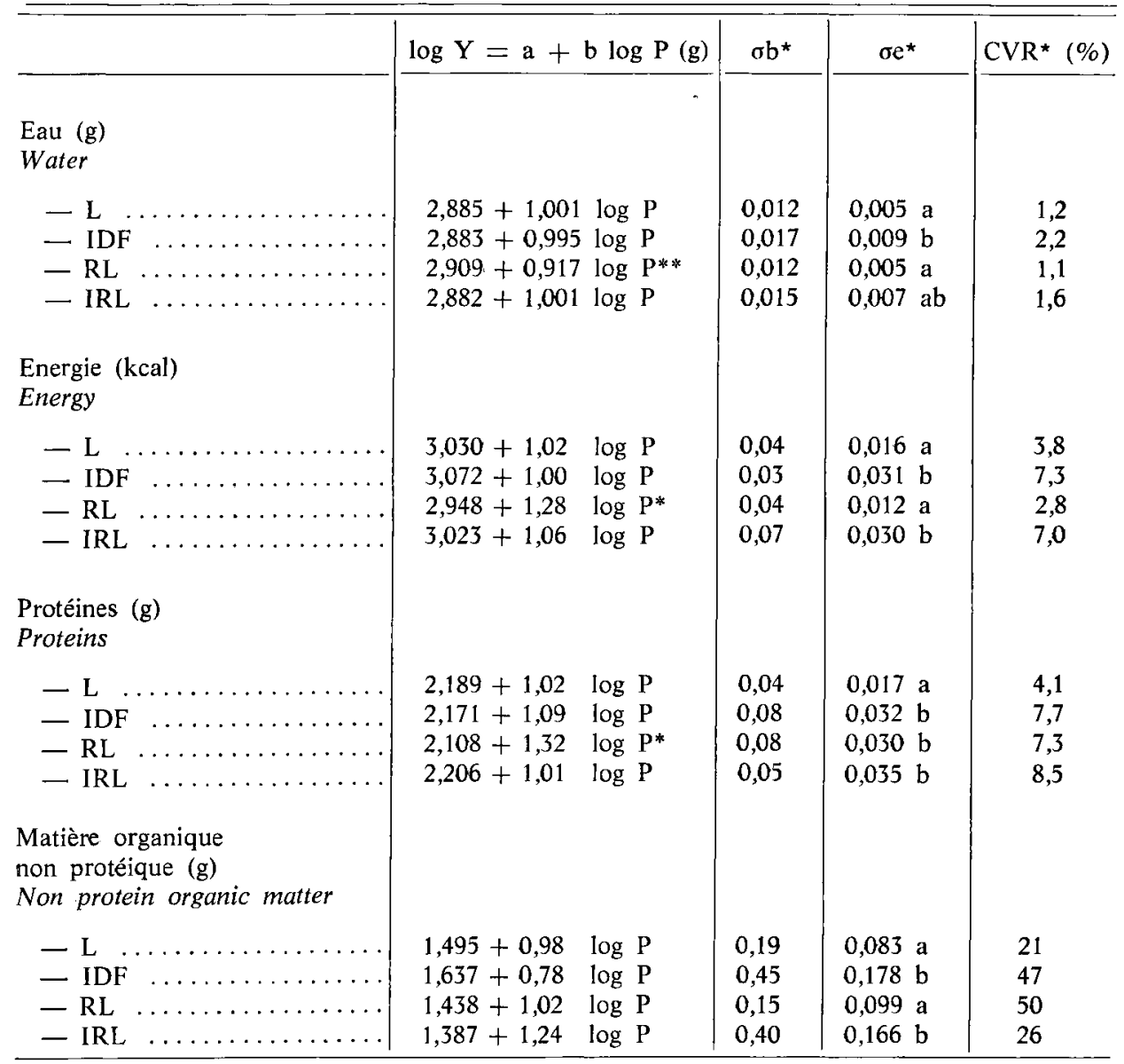

* $\sigma b \quad$ : Ecart-type du coefficient d'allométrie (b). Standard deviation of the allometry coefficient $(b)$.

$\sigma \mathrm{E}:$ Ecart-type résiduel. Ceux ne présentant pas de lettre commune sont significativement $(\mathrm{p}<0,05)$ différents entre eux. Residual standard deviation. Data without common subscripts are significantly $(p<0.05)$ different.

CVR : Coefficient de variation résiduel. Residual variation coefficient.

*,** : Coefficient d'allométrie (b) significativement différent de 1 : à 5 p. $100\left(^{*}\right)$, à 11 p. $100(* *)$ Allometry coefficient (b) significantly different from $1 ; *: 5$ p. $100 ; * *: 1$ p. 100. 


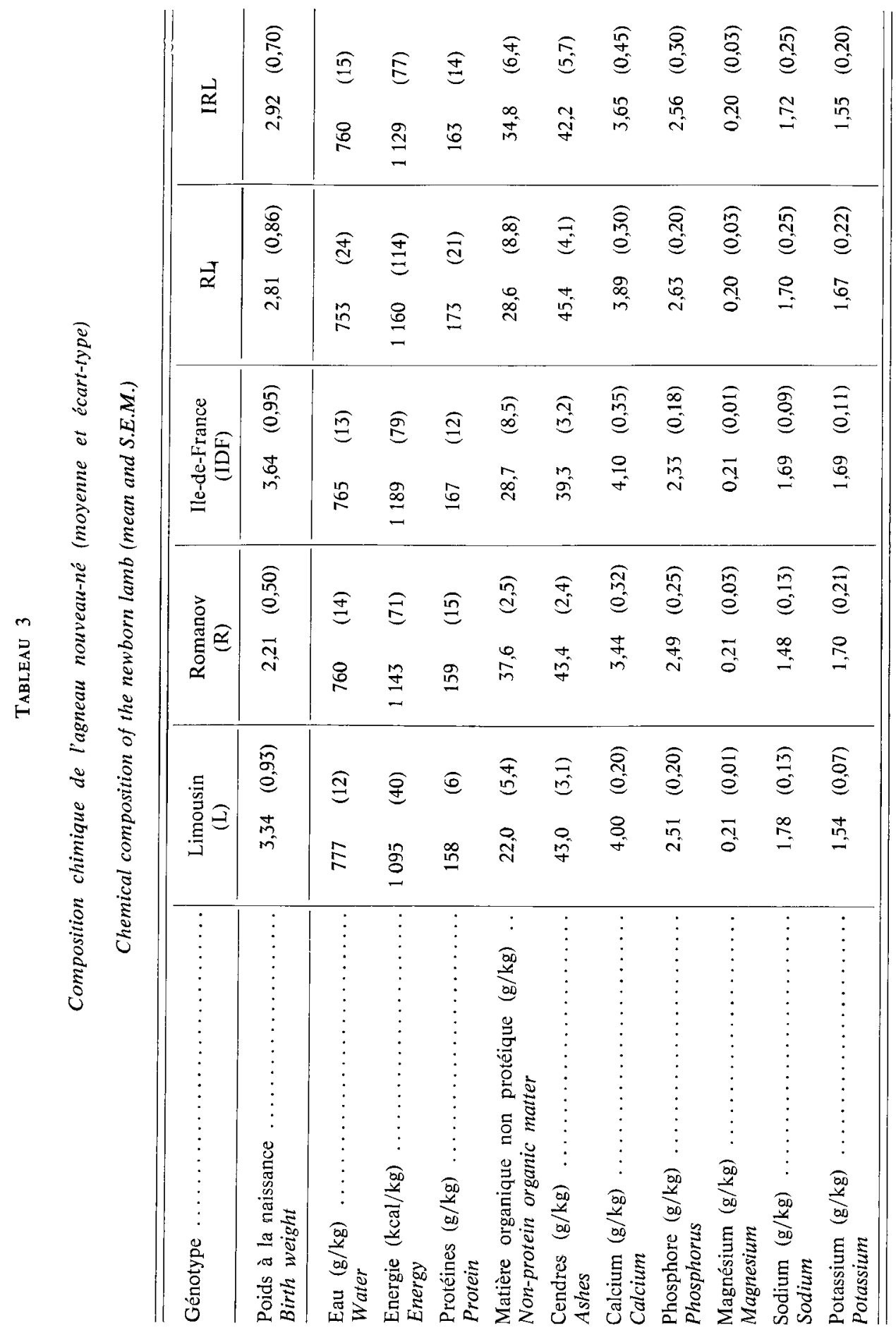


Pour les 3 autres génotypes, les coefficients d'allométrie légèrement supérieurs à 1 ne diffèrent pas significativement entre eux et sont significativement différents ( $p<0,05$ ) de ceux du génotype RL. Pour des plages de variation de poids voisins (tableau 3) les teneurs (Y/P) en énergie ou en protéines des agneaux IDF (1 $189 \mathrm{kcal} /$ $\mathrm{kg}$ et $167 \mathrm{~g} / \mathrm{kg}$ respectivement) sont proches de celles des agneaux IRL (1 $129 \mathrm{kcal} /$ $\mathrm{kg}$ et $163 \mathrm{~g} / \mathrm{kg}$ respectivement) et légèrement supérieures à celles des agneaux de génotype $\mathrm{L}(1095 \mathrm{kcal} / \mathrm{kg}$ et $158 \mathrm{~g} / \mathrm{kg})$. Cependant ces variations intergénotypes testées sur les ordonnées à l'origine (a) des équations d'allométrie ne sont pas significatives.

Enfin, les agneaux de génotype Romanov présentent pour un poids à la naissance nettement plus faible $(2,2 \mathrm{~kg})$ que celui des autres génotypes une teneur moyenne relativement forte en énergie $(1143 \mathrm{kcal} / \mathrm{kg})$ et faible en protéines $(159 \mathrm{~g} /$ $\mathrm{kg}$ ) mais les coefficients de variation sont assez élevés (6 à $10 \mathrm{p}$. 100).

Les résultats obtenus sur la matière organique non protéique sont nettement plus variables (tableau 2). Ainsi on note un coefficient d'allométrie inférieur à 1 pour les agneaux IDF $(0,78)$ proches de 1 pour les agneaux $\mathrm{L}$ et $\mathrm{RL}(0,98$ et 1,02$)$ et supérieur à 1 pour les agneaux IRL $(1,24)$. Cependant aucun coefficient d'allométrie n'est significativement différent de 1 . Les coefficients de variation résiduelle sont élevés (20 à 50 p. 100 ) et les équations de régression ne permettent pas de définir de relation très précise entre la teneur en matière organique non protéique et le poids des agneaux.

Les valeurs moyennes (tableau 3) varient fortement d'un génotype à l'autre de $21,9 \mathrm{~g} / \mathrm{kg}$ (agneaux L) à $37,9 \mathrm{~g} / \mathrm{kg}$ (agneaux Romanov), aussi bien qu'à l'intérieur d'un même génotype, les coefficients de variation étant de l'ordre de 6 à 30 p. 100 .

\section{Teneur en minéraux des agneaux nouveau-nés}

Les relations d'allométrie reliant les quantités totales de cendres au poids à la naissance des agneaux (tableau 4) présentent des coefficients d'allométrie non significativement différents de 1 : légèrement supérieurs à 1 pour les agneaux RL, inférieurs à 1 pour les 3 autres génotypes L, IDF et IRL. En outre ces 4 coefficients ne diffèrent pas entre eux.

Pour les 3 derniers génotypes cités on observe donc une tendance à une diminution de la teneur en cendres ( $\mathrm{Y} / \mathrm{P}$ ) lorsque le poids de naissance augmente (figure 1d) avec des valeurs moyennes variant de 39 à $45 \mathrm{~g} / \mathrm{kg}$ (tableau 3). La comparaison des ordonnées à l'origine (a) n’indique pas de différence significative entre génotypes.

La comparaison des relations d’allométrie reliant les quantités totales de chacun des éléments minéraux analysés (tableau 4) au poids des agneaux révèle quelques différences : Ainsi, le coefficient d'allométrie est significativement supérieur à l'unité dans le cas du magnésium $(b=1,38, p<0,05)$ ou du potassium $(b=1,3, p<0,05)$ chez les agneaux croisés RL. Il est significativement inférieur à l'unité dans le cas du calcium pour les agneaux IRL $(b=0,86, p<0,05)$ et dans le cas du phosphore pour les agneaux $L(b=0,77, p<0,01)$ et $R L(b=0,87, p<0,05)$. Les autres coefficients d'allométrie ne sont pas significativement différents de l'unité, indiquant que le plus souvent les teneurs des différents minéraux $(\mathrm{Y} / \mathrm{P})$ sont indépendantes 


\section{Tableau 4}

Equations d'allométrie reliant des quantités totales $(Y)$ de cendres et des différents minéraux du corps de l'agneau nouveau-né à son poids à la naissance $(P)$

Allometry equations between the total quantities $(Y)$ of ashes and minerals and lamb birth-weight $(P)$

\begin{tabular}{|c|c|c|c|c|}
\hline $\mathrm{Y}$ & $\log Y=a+b \log P(g)$ & $\sigma b^{\star}$ & $\sigma e^{\star}$ & $\begin{array}{c}\text { CVR* } \\
(\%)\end{array}$ \\
\hline \multicolumn{5}{|l|}{ Cendres $(g)$} \\
\hline$-\mathrm{L} \ldots$ & $1,661+0,94 \log P$ & 0,07 & 0,032 a & 7,7 \\
\hline- IDF $\ldots \ldots \ldots \ldots$ & $1,650+0,90 \log \mathrm{P}$ & 0,08 & $0,035 \mathrm{a}$ & 8,4 \\
\hline$-\mathrm{RL} \ldots \ldots \ldots \ldots \ldots$ & $1,620+1,04 \log \mathrm{P}$ & 0,07 & $0,027 \mathrm{~b}$ & 6,3 \\
\hline- IRL $\ldots \ldots \ldots \ldots$ & $1,689+0,85 \log P$ & 0,08 & 0,037 a & 13,0 \\
\hline \multicolumn{5}{|l|}{ Calcium $(\mathrm{g})$} \\
\hline $\begin{array}{c}\text { Calcium } \\
-\mathrm{L} . .\end{array}$ & $1,114+0,94 \log \mathrm{P}$ & 0,08 & 0,022 a & 5,1 \\
\hline - IDF .. & $1,190+0,82 \log \mathrm{P}^{* *}$ & 0,05 & $0,020 \mathrm{a}$ & 4,7 \\
\hline$-\mathrm{RL} \ldots \ldots \ldots \ldots \ldots$ & $1,055+1,03 \log P$ & 0,12 & $0,032 \mathrm{~b}$ & 10,3 \\
\hline$-\operatorname{IRL} \ldots \ldots \ldots \ldots$ & $1,109+0,86 \log \mathrm{P}^{*}$ & 0,05 & $0,035 \mathrm{~b}$ & 8,4 \\
\hline \multicolumn{5}{|l|}{ Phosphore (g) } \\
\hline Phosphorus & & & & \\
\hline $\begin{array}{l}-\mathrm{L} \\
-\mathrm{IDF} \\
\ldots\end{array} \ldots \ldots \ldots \ldots \ldots \ldots$ & $\begin{array}{l}0,830+0,77 \log P^{* *} \\
0,685+0,97 \log P\end{array}$ & $\begin{array}{l}0,08 \\
0,10\end{array}$ & $\begin{array}{l}0,024 \mathrm{a} \\
0,039 \mathrm{~b}\end{array}$ & $\begin{array}{l}5,6 \\
9,3\end{array}$ \\
\hline$-\mathrm{RL} \ldots \ldots \ldots \ldots \ldots$ & $0,775+0,87 \log P^{*}$ & 0,10 & $0,035 \mathrm{~b}$ & 8,5 \\
\hline- IRL $\ldots \ldots \ldots \ldots \ldots$ & $0,711+1,00 \log P$ & 0,06 & $0,036 \mathrm{~b}$ & 8,6 \\
\hline \multicolumn{5}{|l|}{ Magnésium (g) } \\
\hline$-\mathrm{L} \ldots \ldots \ldots \ldots \ldots$ & $-0,716+1,07 \log P$ & 0,06 & 0,018 a & 4,3 \\
\hline- IDF $\ldots \ldots \ldots \ldots \ldots$ & $-0,672+0,98 \log P$ & 0,04 & 0,018 a & 4,1 \\
\hline$-\mathrm{RL} \ldots \ldots \ldots \ldots \ldots$ & $-0,844+1,38 \log \mathrm{P}^{*}$ & 0,16 & $0,053 \mathrm{~b}$ & 13,0 \\
\hline 一 IRL $\ldots \ldots \ldots \ldots \ldots$ & $-0,645+0,86 \log \mathrm{P}^{* *}$ & 0,11 & $0,070 \mathrm{~b}$ & 17,4 \\
\hline \multicolumn{5}{|l|}{ Sodium (g) } \\
\hline$-\mathrm{L} \ldots$ & $0,222+1,05 \log P$ & 0,12 & $0,035 \mathrm{a}$ & 8,4 \\
\hline- IDF $\ldots \ldots \ldots \ldots \ldots$ & $0,300+0,87 \log P^{* *}$ & 0,02 & $0,009 \mathrm{~b}$ & 2,0 \\
\hline$-\mathrm{RL} \ldots \ldots \ldots \ldots \ldots \ldots$ & $0,238+0,95 \log P$ & 0,22 & $0,072 \mathrm{c}$ & 18,0 \\
\hline$-\operatorname{IRL} \ldots \ldots \ldots \ldots \ldots$ & $0,287+0,90 \log P$ & 0,07 & 0,046 a & 11,4 \\
\hline \multicolumn{5}{|l|}{ Potassium (g) } \\
\hline Potassium & & & & \\
\hline $\begin{array}{l}-\mathrm{L} \\
-\mathrm{IDF}\end{array}$ & $0,215+0,95 \log P$ & 0,07 & 0,020 a & 4,7 \\
\hline $\begin{array}{l}- \text { IDF } \\
-\mathrm{RL} .\end{array}$ & $0,175+1,09 \log \mathrm{P}$ & 0,07 & 0,027 a & $\begin{array}{r}4,0 \\
1,0\end{array}$ \\
\hline $\begin{array}{l}\text { - RL } \\
\text { - IRL }\end{array}$ & $0,210+0,96 \log P$ & 0,07 & $0,027 \mathrm{a}$ & $\begin{array}{r}12,0 \\
6,0\end{array}$ \\
\hline
\end{tabular}

* $\sigma \mathrm{b}$ : Ecart-type du coefficient d'allométrie (b).

Standard deviation of the allometry coefficient $(b)$.

$\sigma \mathrm{E}:$ Ecart-type résiduel. Ceux ne présentant pas de lettre commune sont significativement $(\mathrm{p}<0,05)$ différents entre eux.

Residual standard deviation. Data without common subscripts are significantly $(p<0.05)$ different.

CVR : Coefficient de variation résiduel. Residual variation coefficient.

***: Coefficient d'allométrie (b) significativement différent de 1 : à 5 p. 100 (*), à 1 p. $100(* *)$. Allometry coefficient (b) significantly different from $1 ; *: 5$ p. $100 ; * *: 1$ p. 100. 
du poids à la naissance. Les valeurs moyennes observées sont proches pour tous les génotypes (tableau 3).

\section{4. - Discussion}

La composition chimique moyenne des agneaux des 5 génotypes étudiés (teneurs en eau de 753 à $777 \mathrm{~g} / \mathrm{kg}$, teneurs en énergie de 1129 à $1189 \mathrm{kcal} / \mathrm{kg}$, teneurs en protéines de 158 à $173 \mathrm{~g} / \mathrm{kg}$, teneurs en cendres de 42,2 à 45,4 g/ kg) est assez proche de celles décrites antérieurement pour des agneaux nouveau-nés (Mirchell, Kammalade \& Hamilton, 1928, Weninger, Funk \& Koenig, 1955, Langlands \& Sutherland, 1969, Searle, 1970, Sykes \& Field, 1972) et représentées dans les figures $1 \mathrm{~b}$ à $1 \mathrm{~d}$. Il en est de même pour la composition minérale et nos résultats sont en accord avec ceux de Sykes \& Dingwall (1976) si ce n'est pour les teneurs en sodium légèrement plus élevées dans leur étude.

En revanche ces résultats se différencient nettement de la composition de fœetus proches du terme issus de croisements avec des béliers Suffolk (RATTRAY et al., 1974, Mac Donald et al., 1979, figure 1 à $1 \mathrm{~d}$ ), plus riches en eau (780 à $817 \mathrm{~g} / \mathrm{kg}$ ) mais moins riches en énergie (900 à $1050 \mathrm{kcal} / \mathrm{kg})$, en protéines $(125$ à $145 \mathrm{~g} / \mathrm{kg}$ ) ou en cendres (33 à $35 \mathrm{~g} / \mathrm{kg}$ ). Trois hypothèses peuvent expliquer ces différences de composition entre fotus proches du terme et nouveau-nés :

- les différences pourraient être liées au génotype des animaux;

- les processus de maturation des fotus en fin de gestation pourraient s'accompagner d'une diminution de leur teneur en eau;

- le degré d'humidification de la toison des fœetus baignant dans le liquide amniotique doit être vraisemblablement supérieur à celui d'agneaux nouveau-nés ayant passé quelques heures à l'air libre. Ainsi, la teneur en eau d'agneaux nouveaunés léchés par leur mère n'est plus que de $700 \mathrm{~g} / \mathrm{kg}$ (Sykes \& FIELD, 1972).

A partir de l'ensemble des données rapportées ci-dessus et de celles disponibles dans la littérature et ayant été réexploitées par l'A.R.C. (1980) (figures 1a et 1c), l'étude des liaisons entre le poids à la naissance des agneaux et leur composition chimique permet de distinguer deux groupes de résultats : a) pour 3 des génotypes que nous avons étudiés ( $L, I D F$ et IRL) les teneurs en eau, énergie et protéines du corps des animaux sont indépendantes de leur poids. Il en est de même dans le cas d'agneaux Rambouillet et Southdown (Mitchell, Kammalade \& Hamilton, 1928) et d'agneaux simples Scottish Blackface (Sykes \& FiEld, 1972). En revanche, b) pour les agneaux croisés RL la teneur en eau diminue tandis que celles en protéines et en énergie augmentent quand le poids à la naissance augmente. Des résultats similaires sont observés pour des agneaux Mérinos (Langland \& SutHERLAND, 1969; Searle, 1970), pour des agneaux issus de portées doubles Scottish Blackface (SYKes \& FIELD, 1972) ainsi que pour des fotus proches du terme (Mac DONAlD et al., 1979). Ce deuxième groupe de résultats est en accord avec ceux de Wallace (1948) et d'Alexander (1974) : Pour ces auteurs une diminution du poids à la naissance s'accompagne d'une diminution des proportions des tissus musculaires et surtout adipeux au bénéfice des proportions de tissus nerveux et osseux. Cependant, dans ces expériences, les variations de poids des agneaux étaient essen- 

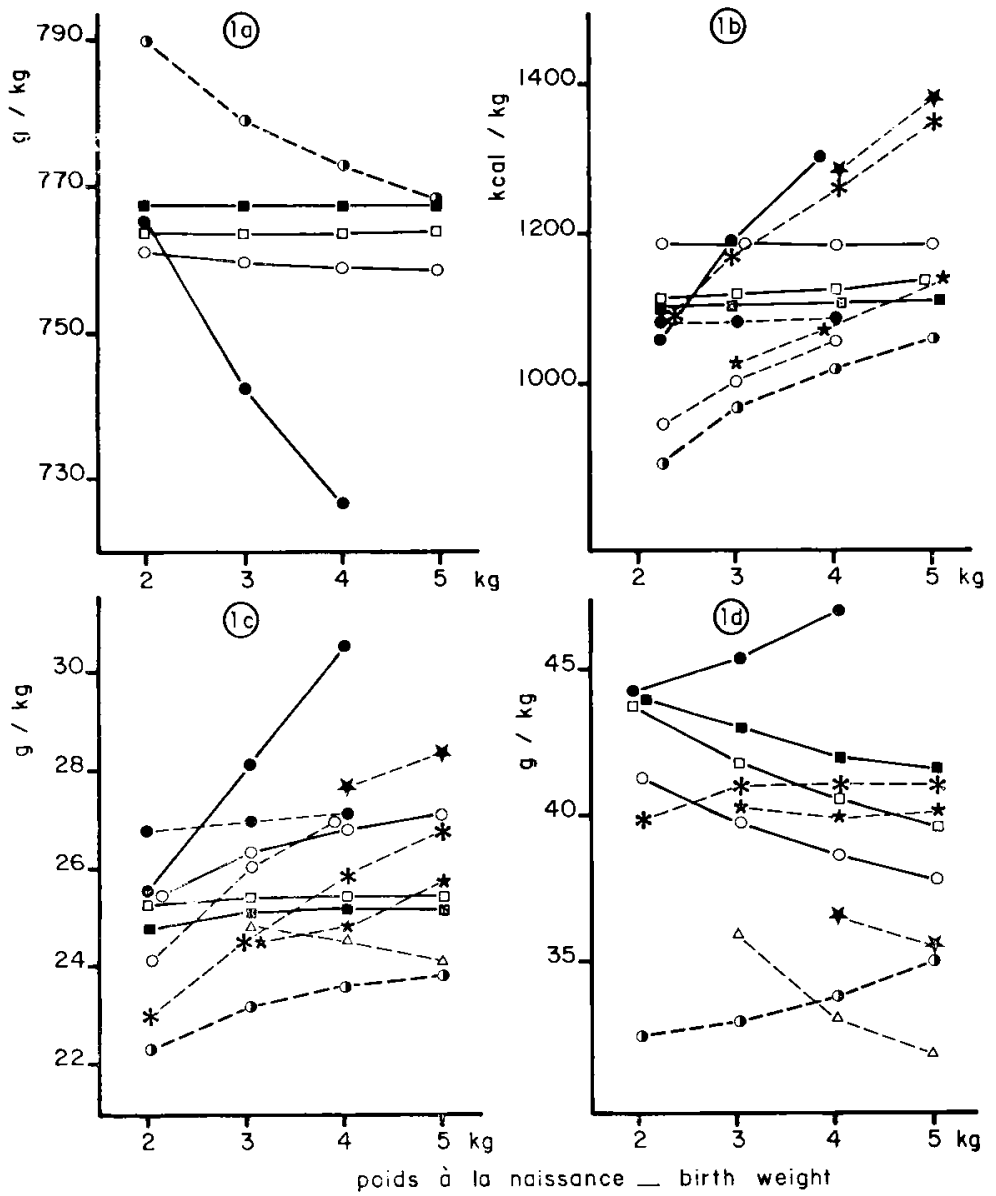

FIG. la à ld

Influence du poids à la naissance sur les teneurs en eau (a), énergie (b), protéines (c) et cendres (d) d'agneaux nouveau-nés ou de foctus à terme

Influence of birthweight on water (a), energy (b), protein (c) and ash (d) content of the new-born lamb

Données de l'étude présente estimées à partir des relations d'allométrie $\mathbf{Z}=\mathbf{L}$,

- = RL, $\square=\mathrm{IRL}, \mathrm{O}=\mathrm{I}$.

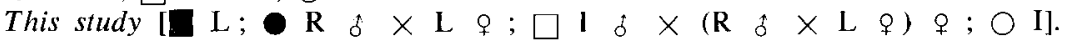

Données bibliographiques sur agneaux nouveau-nés (ARC, 1980).

Available data concerning newborn lambs as summed up by (ARC, 1980).

Scottish Blackface nés doubles (twins) et Scottish Blackface nés simples (singles) (Sykes et Field, 1972).

* Mérinos et Karakul (Weninger, Funk et Kenig, 1955).

$\triangle$ Rambouillet and Southdown (Mitchell et al., 1928); * Merinos (LANGLANDS et Sutherland, 1969 ; $\star$ Mérinos (Searle, 1970).

- - - Fctus proches du terme (145 jours) croisés Suffolk (Mac Donald et al., 1979).

Full term foetuses Suffolk $\delta \times$ (Finnish landrace $\delta \times$ Dorset Horn $q) ~ q$ by Mac Donald and al., 1979. 
tiellement liées à celles des niveaux d’alimentation des brebis gestantes tandis que dans notre étude l'origine des variations du poids des agneaux à la naissance est tout à fait aléatoire et vraisemblablement multiple. Or WIDDowson (1974) observe chez le cobaye nouveau-né que de cette origine dépendent les variations de la teneur corporelle en lipides.

Les travaux d'Alexander (1974) sur les mécanismes de la thermorégulation de l'agneau nouveau-né ont permis d'expliquer l'accroissement de la mortalité néonatale suite à une diminution du poids à la naissance, d’une part par laugmentation des pertes de chaleur et d'autre part par la diminution des réserves énergétiques. Ce dernier résultat ne semble pas s appliquer à la comparaison de la vitalité d’agneaux nouveau-nés de différents génotypes. En effet, pour des tailles de portées voisines, les agneaux IDF et $\mathrm{L}$ présentent des taux de mortalité avant sevrage $(20,1$ p. 100 et 13 p. 100 respectivement) supérieurs à celui des agneaux RL (9,5 p. 100) (Houssin-Villette \& Brelurut, 1980). Or d'après les résultats rapportés ci-dessus, les quantités totales en matière organique non protéique, c'est-à-dire essentiellement en lipides et en glycogène sont voisines d'un génotype à l'autre. Exprimées en énergie ces réserves sont légèrement supérieures pour les agneaux IDF $(253 \mathrm{kcal} / \mathrm{g})$ et $\mathbf{L}(210 \mathrm{kcal} / \mathrm{kg})$ comparées à celles des agneaux RL $(191 \mathrm{kcal} / \mathrm{kg})$. Ainsi des variations quantitatives en réserves énergétiques ne peuvent expliquer les différences de mortalité observées entre génotypes. En revanche, la composition de ces réserves énergétiques non protéiques varie avec le génotype. En effet, si lon prend une valeur énergétique des protéines de $5,6 \mathrm{kcal} / \mathrm{g}$, la matière organique non protéique des agneaux IDF et $\mathrm{L}$ semble principalement constituée de lipides puisque sa teneur en énergie est de 8,4 ou $8,9 \mathrm{kcal} / \mathrm{kg}$, alors que chez les agneaux RL elle comporte seulement $6,1 \mathrm{kcal} / \mathrm{g}$, en raison, selon toute vraisemblance, de l'accroissement des proportions de glycogène. De même les agneaux Romanov pur ou IRL présentent des teneurs en énergie de la matière organique non protéique voisine des agneaux $\mathrm{RL}(6,2 \mathrm{kcal} / \mathrm{g}$ et 5,8 respectivement). Ces différences dans la nature des réserves énergétiques sont peut-être à relier à la meilleure viabilité des agneaux issus de parents Romanov ou croisés Romanov (Ricordeau et al., 1977).

Accepté pour publication en juin 1981.

\section{Remerciements}

A Marie-Jeanne Davicco, pour les dosages des différents minéraux.

\section{Summary}

Influence of birthweight and genotype on the chemical composition of newborn lambs

The chemical composition of 76 newborn lambs from different breeds 114 Limousin (L), 5 Romanov (R) and 12 Ile-de-France (IDF)] or their crosses $[9 \mathrm{R} \& \times \mathrm{L}$ \% (RL); 36 IDF $\delta \times \mathrm{RL} ;$ (IRL)] was determined and related to birth weight except for Romanov (tabl. 1 and 3 ). 
For the three genotypes (L, IDF and IRL) water $(765 \pm 14 \mathrm{~g} / \mathrm{kg}$ ), energy $(1133$ $\pm 70 \mathrm{~g} / \mathrm{g})$ and protein $(163 \pm 13 \mathrm{~g} / \mathrm{kg})$ contents of the lambs were not related to birthweigth. However, for genotype RL, the allometry coefficients were significantly different from I : $0.92(\mathrm{P}<0.01), 1.28(\mathrm{P}<0.05)$ and $1.32(\mathrm{P}<0.05)$, respectively (tabl. 2$)$.

Contents of ash $(42.7 \pm 4.7 \mathrm{~g} / \mathrm{kg}$ ) and minerals (calcium, phosphorus, magnesium, sodium and potassium) were not related to birthweight.

These results on the chemical composition of newborn lambs are discussed in relation with the neonatal mortality rate observed in the same genotypes.

\section{Références bibliographiques}

Alexander G., 1974. Birthweight of lambs : influences and consequences. In Size at birth. Ciba fundation Symposium, 27, 215-239.

A.R.C., 1980. The nutrients requirements of Ruminant livestock. Publication du Commonwealth Agricultural Bureaux.

Houssin-Villette Y., Brelurut A., 1980. Mortalité avant le sevrage d'Agneaux de différents génotypes dans un troupeau en conduite intensive. Bull. tcch. CRZV Theix, I.N.R.A., 40, $5-12$.

I.N.R.A., 1978. In «Alimentation des ruminants». Reproduction, gestation, lactation, 229-243, éd. I.N.R.A. publications, Versailles.

Kalckar H.M., 1947. The enzymatic synthesis of purine ribosides. J. biol. Chem., 167, 477-486.

Langlands J.P., Sutherland H.A.M., 1968. An estimate of the nutrients utilized for pregnancy by Merino sheep. Br. J. Nutr., 22, 217-227.

Langlands J.P., Sutherland H.A.M., 1969. An estimate of the nutrients utilized for live weight gain by Merino sheep. Br.J. Nutr., 23, 603-609.

Lodge G.A., Heanney D.P., 1973. Composition of weight change in the pregnant ewe. J. anin. Sci., 53, 95-105.

Mac Donald I., Robinson J.J., Fraser C., Smart R.I., 1979. Studies on reproduction in prolific ewes. 5. - Accretion of nutrient in the foetuses and adnexa. J. agric. Sci. Camb., 92. 591-603.

Mac Donald I., Wehnham G., Robinson J.J., 1977. Studies on reproduction in prolific ewes. 3. - The development of size and shape of the foetal skeleton. J. agric. Sci. Camb., 85, $373-391$.

Mitchell H.H., Kammalade W.G., Hamilton T.S., 1928. Bull. ill. agric. exp. sfa. (cité dans A.R.C., 1980).

Rattray P.V., Garrett W.N., East N.E., Hinman N., 1974. Growth, development and composition of the ovine conceptus and mammary gland during pregnancy. J. amim. Sci., 38, $613-626$.

Ricordeau G., Tchamitchian L., Lebevre C., Brunel J.C., 1977. Amélioration de la productivité des Brebis Berrichonnes du Cher par croisement. IV. - Durée de gestation et viabilité des Agneaux Berrichons, Romanov et croisés F1, F2, F3. Ann. Génét. Súl. anim., 9, 219-239.

SEARLE T.W., 1970. Body composition on lambs and young sheep and its prediction in vivo from tritiated water space and body weight. J. agric. Sci. Camb., 74, 357-362.

Sykes A.R., Dingwall R.A., 1976. The phosphorus requirement of pregnant sheep. J. agric. Sci. Camb., 86, 587-594. 
Sykes A.R., Field A.C., 1972. Effect of dietary deficiency of energy protein and calcium in the pregnant ewe. II. - Body composition and mineral content of the lamb. J. agric. Sci. Camb., 78, 119-125.

Tissier M., Theriez M., 1978. Influence du niveau des apports énergétiques distribués à la Brebis pendant la gestation sur le poids de naissance et la croissance des agneaux. Ann. Biol. anim. Biochim. Biophys., 19, 235-240.

WALlace L.R., 1948. The growth of lambs before and after birth in relation to the level of nutrition. J. agric. Sci. Camb., 38, 93-153.

Weninger J.H., Funk K., Kenig K.H., 1955. Untersuchungen über den Calcium, Phosphor und Nährstoffgehaet von Schafen und Ziegen. Arch. Tierernähr., 5, $216-224$.

WidDowson E.M., 1974. Immediate and long-term consequences of being large or small at birth : a comparative approach. Ciha fundation Symposium, 27, 65-76. 